\title{
Characterization of the Photoluminescence of the Red Alga Gelidium amansii
}

\author{
Han Joo Lee ${ }^{1}$, Sang Mok Jung ${ }^{1}$, Han Seong Lee ${ }^{2}$, SeulGi Kang ${ }^{1}$, Ji Su Son ${ }^{1}$, Jae Hyuk Jeon ${ }^{1}$, \\ Hyun Woung Shin ${ }^{1}$
}

${ }^{1}$ Department of Life Science and Biotechnology, Soonchunhyang University, Asan-si, South Korea

${ }^{2}$ Department of Forensic Investigation, Research Center of National Coast Guard, Korea

\section{Email address:}

happynews4me@gmail.com (H. W. Shin), thinkdi@gmail.com (S. M. Jung)

\section{To cite this article:}

Han JooLee, Sang Mok Jung, Han Seong Lee, SeulGi Kang, Ji Su Son, Jae Hyuk Jeon, Hyun Woung Shin. Characterization of the Photoluminescence of the Red Alga Gelidium amansii. European Journal of Biophysics. Vol. 3, No. 2, 2015, pp. 14-18.

doi: $10.11648 /$ j.ejb.20150302.12

\begin{abstract}
Naturally occurring substances have been used increasingly for a number of applications, with advantages such as their low cost, ecofriendliness, and renewability. This study investigated natural substances that may be used in organic light-emitting diodes (OLEDs). An extract of the marine macroalga Gelidium amansii was fractionated using column chromatography. The photoluminescence activity of the fractions showed peaks at 670-680 nm and Fourier transform infrared (FT-IR) spectroscopy and ${ }^{1} \mathrm{H}$ nuclear magnetic resonance (NMR) analysis identified the photoactive compound as violaxanthin.
\end{abstract}

Keywords: Gelidium amansii, OLED, Violaxanthin

\section{Introduction}

The development of organic light-emitting diodes (OLEDs) for use in displays has progressed markedly, as they have excellent performance characteristics, such as fast response times and a wide viewing angle in a single structure (Geffroy et al., 2006). The fabrication of OLEDs uses double-charge injection devices, requiring the simultaneous supply of both electrons and holes to organic materials (Wong and Ho, 2009).In organic materials, electrons and holes recombine to form excitons, which emit light at a characteristic frequency based on the energy difference between the highest (HOMO) and lowest (LUMO) occupied molecular orbitals of the organic material (Yersin, 2004). However, the transport of the electrons and holes to the emitting organic layer can be difficult. Additional layers are needed to promote transport, such as a transparent conducting oxide layer, hole transport layer (HTL), electron transport layer (ETL), emitting layer (EL), electron blocking layer (EBL), and hole blocking layer (HBL) (Shoustickov et al, 1998). Tris(8-hydroxyquinoline)aluminum(III) (Alq3) and 1,2,3,4,5-pentaphenyl-1,3-cyclopentadiene (PPCP) have been used widely in OLEDs as ETLs and ELs (Odaka et al., 2006; Zhao et al., 2009). HTLs are composed of N,N'-diphenyl-N,N'-bis(1-naphthyl)-1,1'-biphenyl-4,4"-diam ine

(NPB)

and

N,N'-bis(3-methylphenyl)-N,N'-diphenyl-[1,1'-biphenyl]-4,4' -(diamine) (TPD) (Popovic et al., 2002). The polymer used in polymer light-emitting diodes (PLED) is synthesized from derivatives of poly(p-phenylenevinylene) and polyfluorene (Wu et al., 1995). The advantages of using polymers include increased performance and easy manufacture. Recently, OLED devices using polysilicon thin film transistors (TFTs) have demonstrated potential for better image quality and lower power consumption; however, improvements are required to lighting performance and color durability for displays, and these devices still suffer from a limited lifespan, water damage, and low electron and hole carrier efficiencies (Kamiya et al., 2009) In addition, there are limitations to developing a synthesis process, especially high cost and pollution (Cho et al., 2009).An alternative synthesis method that uses living organisms instead of chemical synthesis has been developed. Gomez et al. (2014a) investigated integrating deoxyribonucleic acid (DNA), ribonucleic acid (RNA), and their nucleobases into OLEDs to improve efficiency. Marine organisms such as bacteria, fungi, seaweeds, and animals have been used successfully in bioactive applications such as in medicine, food, and energy (Holmstrom and Kjelleberg, 1999).This study used a solvent extraction process to obtain compounds from the marine red 
alga Gelidium amansii for integration with OLEDs. The extraction fractions were characterizedusing photoluminescence (PL) and analyzed chemically by Fourier transform infrared (FT-IR) spectroscopy and proton nuclear magnetic resonance $\left({ }^{1} \mathrm{H}-\mathrm{NMR}\right)$ findings.

\section{Materials and Methods}

\subsection{Collection and Extraction}

A marine macroalga, Gelidium amansii, was collected from a harbor in Guryungpo, Pohang, Gyeongsangnam-do, Korea (35 $\left.59^{\prime} 41.06^{\prime \prime}, 129^{\circ} 33^{\prime} 59.73^{\prime \prime}\right)$ (Figure 1) and transported immediately to the laboratory. The samples were washed with filtered seawater to remove epiphytes and the resulting materials were dried at room temperature. Then, 1 $\mathrm{kg}$ of dried material was crushed to a fine powder to increase the extraction rate. The material was extracted in methanol with the total volume made up to $10 \mathrm{~L}$, for $24 \mathrm{~h}$ at room temperature. The supernatant was filtered through a $0.22-\mu \mathrm{m}$ filter and kept at $20^{\circ} \mathrm{C}$. The extracted solution was vacuum-evaporated at $37^{\circ} \mathrm{C}$.

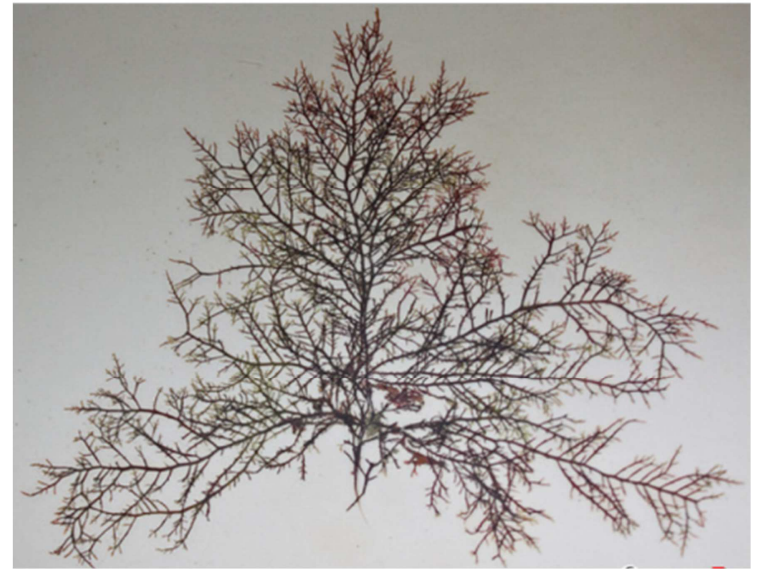

Figure 1. Illustration of Gelidiumamansii.

\subsection{Isolation and Photoluminescence}

To isolate mono spots, thin layer chromatography (TLC) was performed using hexane: acetone in a ratio of 70:30, and the spots were identified with ultraviolet (UV) light at wavelength $365 \mathrm{~nm}$ (SILG/UV254, $0.25 \mathrm{~mm}$ layer with fluorescent indicator, Macherey-Nagel, Germany). The active spot was fractionated using column chromatography, with the stationary phase in silica gel $(60 \AA$, sigma) and the mobile phase in organic solvent (70:30 hexane: acetone). The eluent was collected in a series of fractions taken every $20 \mathrm{~mL}$ and then labeled. The PL spectrum of all fractions was measured. The fractions were exposed to UV light at $365 \mathrm{~nm}$ in a $1 \mathrm{~mL}$ crystal cuvette, which caused a PL reaction that was assessed using a Minolta spectroradiometer (CS-1000, Japan).

\subsection{Analysis}

The Fourier transform infrared (FT-IR) spectrum of fraction number 15-2 was recorded. Samples were prepared on a
KBrdisk under a hydrostatic press with a force of $5.2 \mathrm{~T} / \mathrm{cm}^{2}$ for $3 \mathrm{~min}$. The scanning range was $450-4000 \mathrm{~cm}^{-1}$ and the resolution was $1 \mathrm{~cm}^{-1}$.The ${ }^{1} \mathrm{H}-\mathrm{NMR}(400 \mathrm{MHz})$ spectra were analyzed at room conditions using an ARX-400 spectrometer (Billerica, MA, USA) with CDC13 solvent.

\section{Results}

\subsection{Isolation and Purification}

The active extract was separated using TLC. Three spots were identified, with retention factor ( $\mathrm{rf}$ ) values of 0.107 , 0.178 , and 0.321 , respectively (Figure 2). The spot with an rf of 0.107 showed the highest activity. This strong active spot was purified sequentially by silica gel column chromatography.

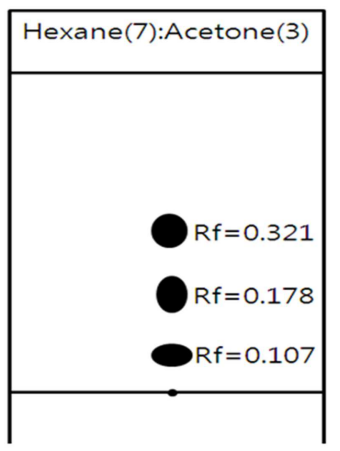

Figure 2. Analysis of thin layer chromatography from Gelidiumamansiiextract.

The PL intensities of the purified fractions had a maximum wavelength of $670-680 \mathrm{~nm}$. Fraction number $15-2$ had the highest intensity, at $0.0035 \mathrm{~W} / \mathrm{Sr} \mathrm{m}^{2}$, and fraction number $23-2$ had the lowest recorded intensity (Figure 3 ). The measured PL activity of the fractions was in the order 15-2 $>20-2>20-1>$ $23-1>15-1>22-2>23-2$.

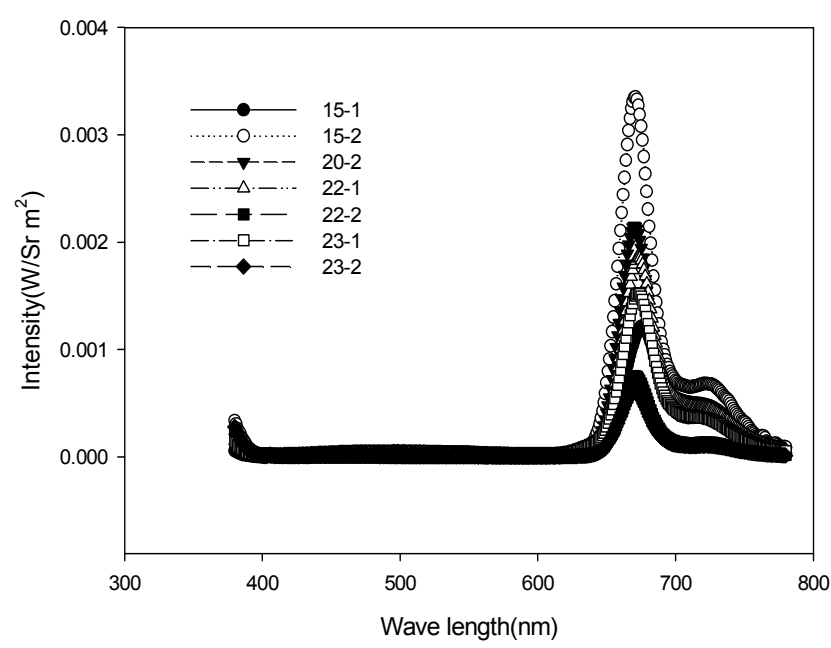

Figure 3. PL spectrum of selected fraction from Gelidiumamansiiextract.

\subsection{IR and ${ }^{1} \mathrm{H}$ NMR Analysis}

The FT-IR spectrum showed a strong peak at $3400 \mathrm{~cm}^{-1}$ (Figure 4), related to the hydroxyl group. The peak 
between 3200 and $2800 \mathrm{~cm}^{-1}$ was due to a long carbon chain. The weak peak located at $1680 \mathrm{~cm}^{-1}$ indicated a long chain of double bonded carbon $(\mathrm{C}=\mathrm{C})$. The aromatic function can be identified at 1597,1520 , and $1474 \mathrm{~cm}^{-1}$.

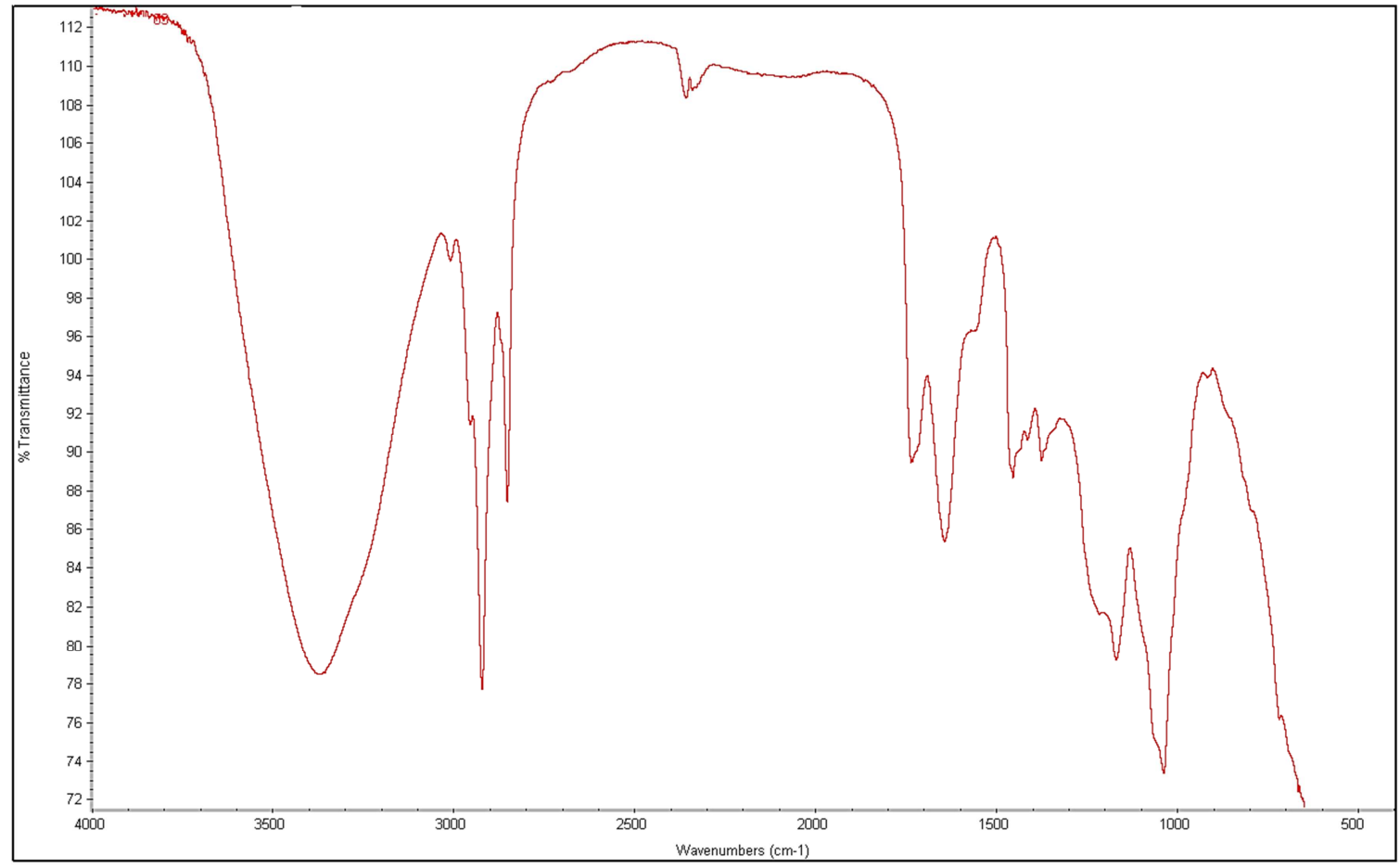

Figure 4. Analysis of IR fraction from Gelidiumamansiiextract.

${ }^{1} \mathrm{H}-\mathrm{NMR}$ resulted in a proton spectrum in which all of the molecules were in the range $7.9-4.8 \mathrm{ppm}$ (Figure 5). The resonances at $7.1 \pm 0.05 \mathrm{ppm}$ contained the H12', H14', H14, and $\mathrm{H} 10$ signals. The sample was identified as violaxanthin based on the FT-IR and ${ }^{1} \mathrm{H}-\mathrm{NMR}$ findings (Figure 6).

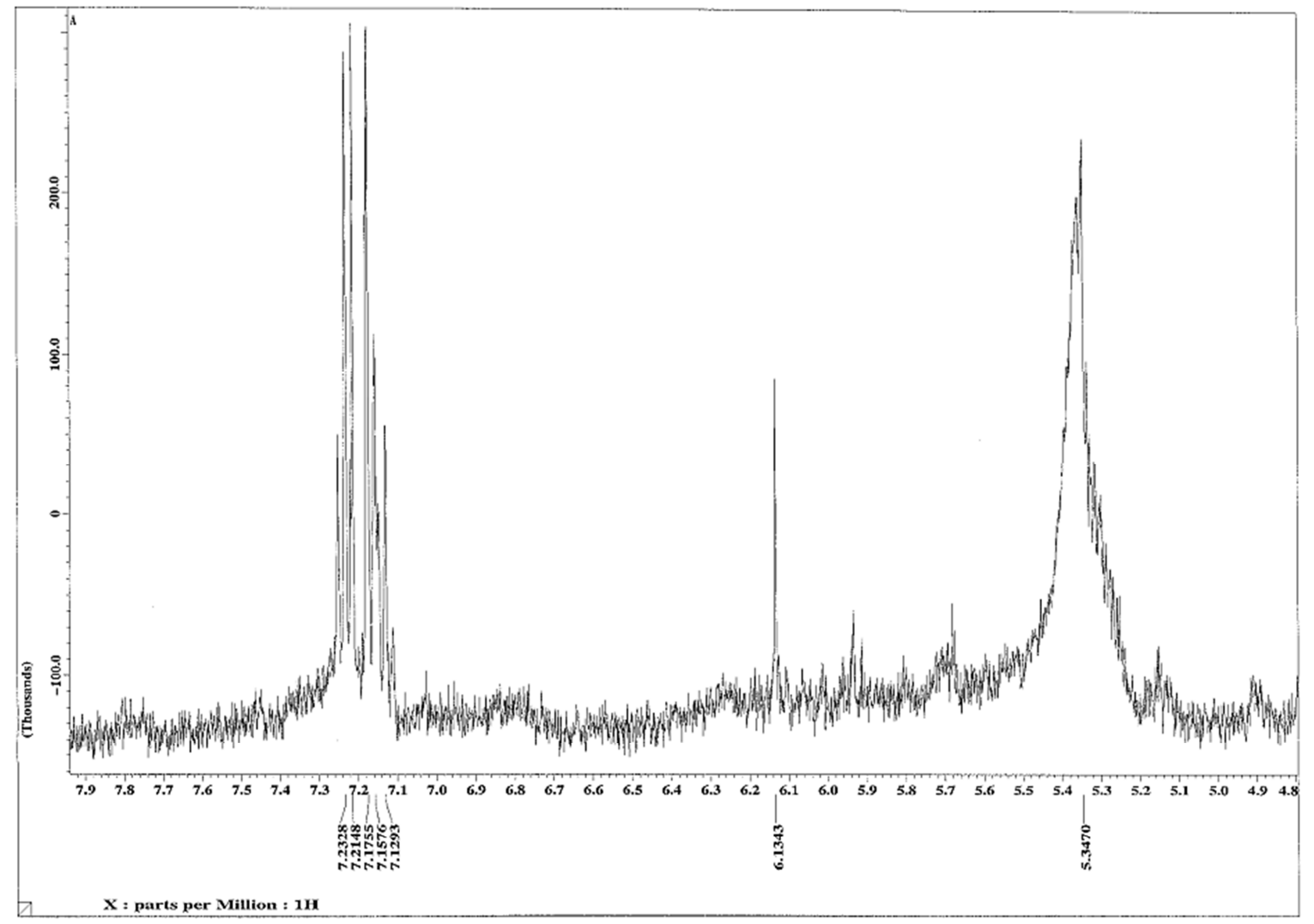

Figure 5. 1H-NMR analysis of a fraction from Gelidiumamansii extract. 


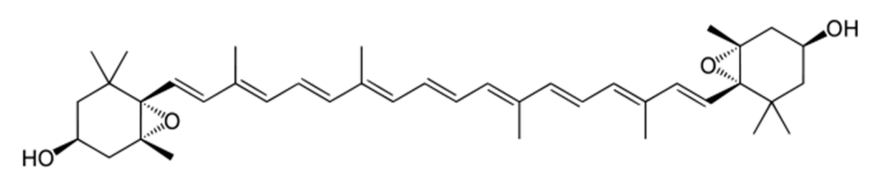

Figure 6. Chemical structure of violaxanthin from Gelidiumamansii.

\section{Discussion}

Living organisms provide useful resources and bioactive compounds have been used extensively in medicines and food, and their high carbohydrate content makes them suitable for use as bioenergy resources (Wi et al., 2009).The marine macroalga Gelidium amansii is an important species economically and is used in the phytochemical industry for the production of agar powder, antioxidants, and chemical reagents (Liu et al., 2004). Numata et al. (1991) reported that an extract of Gelidium amansii inhibited cell growth. Marine macroalgae contain pigments with distinct optical characteristics, such as phycobilins, anthocyanins, betalains, and chlorophylls, which deliver electrons from light energy or metabolic processes (Hader and Figueroa, 1997). The nucleic bases adenine, guanine, cytosine, and thymine, extracted from living organisms, have been inserted into multilayered OLED devices; in particular, adenine and cytosine have yielded significant improvements in electron transport in these systems (Gomez et al., 2014b). The electroluminescence (EL) quality of OLEDs is strongly correlated with the photoluminescence (PL) activity (Seo and Moon, 2008; Winter et al., 2008). This paper investigated a photoluminescent compound extracted from Gelidium amansii. The algal extract was fractionated using column chromatography and the resulting fractions were examined using PL spectroscopy. Some fractions showed high PL intensity at wavelengths of $650-680 \mathrm{~nm}$. FT-IR and $\mathrm{H}^{1} \mathrm{NMR}$ analyses confirmed that the extracted fraction was a violaxanthin, a natural pigment in plant metabolites related to the photosynthetic system, where it both harvests light energy and protects against excess light energy (Havaux and Niyogi, 1999). In the light-emitting layer of an OLED, the recombination of electrons and holes results in an excited state, which ultimately emits light via a singlet or triplet state pathway. Photosynthesis also produces singlet or triplet states in the chloroplasts of plants (Demming-Adams and Adams, 1992). Shimatani et al. (2005) reported that OLEDs fabricated with chlorophyll, another photosynthetic pigment, exhibited an EL and PL spectrum at wavelengths of 700-750 nm.The results suggest that this extract can act as an electron carrier in both biological systems and OLEDs. There are many advantages to using natural substances, since they are renewable, inexpensive, and ecofriendly and result in enhanced performance. Several candidates should be studied further to improve their emission efficiencies.

\section{Acknowledgement}

This research was supported by the Basic Core Technology
Development Program for the Ocean and the Polar Regions of the National Research Foundation (NRF) funded by the Ministry of Science, ICT \& Future Planning (2010-0020711).

\section{References}

[1] Cho DH, Yang S, Ko SH, Park CB, Yoon SM, Lee JI, Hwang CS, Chu HY and Cho KI (2009) 21.2: Al and Sn-Doped Zinc Indium Oxide Thin Film Transistors for AMOLED Back-Plane. In SID Symposium Digest of Technical Papers (Vol. 40, No. 1, pp. 280-283). Blackwell Publishing Ltd.

[2] Demmig-Adams B and Adams Iii WW (1992) Photoprotection and other responses of plants to high light stress. Annu. Rev. Plant Biol.43(1): 599-626.

[3] Kamiya T, Kenji N and Hideo H (2009) Origins of high mobility and low operation voltage of amorphous oxide TFTs: electronic structure, electron transport, defects and doping. $\mathrm{J}$ Disp. Technol. 5(12): 468-483.

[4] Geffroy B, Le Roy P and Prat C. (2006) Organic light-emitting diode (OLED) technology: materials, devices and display technologies. Polym. INT.55(6): 572-582.

[5] Gomez EF, Venkatraman V, Grote JG and Steckl AJ (2014a) DNA Bases thymine and adenine in bio-organic light emitting diodes. Sci. Rep. 4: 1-5

[6] Gomez EF, Venkatraman V, Grote JG and Steckl AJ (2014b) Exploring the potential of nucleic acid bases in organic light emitting diodes. Adv. Mater. 1-4.

[7] Havaux M and Niyogi KK (1999) Theviolaxanthin cycle protects plants from photooxidative damage by more than one mechanism. Proceedings of the National Academy of Sciences. 96(15): 8762-8767.

[8] Holmström C and Kjelleberg S (1999) Marine Pseudoalteromonas species are associated with higher organisms and produce biologically active extracellular agents. FEMS Microbiol. Ecol.30(4): 285-293.

[9] Häder DP and Figueroa FL (1997) Photoecophysiology of marine macroalgae. Photochem. Photobiol. 66(1): 1-14.

[10] Liu D, Amy P and Sun J (2004) Preliminary study on the responses of three marine algae, Ulva pertusa (Chlorophyta), Gelidium amansii (Rhodophyta) and Sargassumenerve (Phaeophyta), to nitrogen source and its availability. J. Ocean Univ. China.3(1): 75-79.

[11] Numata A, Kanbara S, Takahashi C, Fujiki R, Yoneda M, Fujita E and Nabeshima Y (1991) Cytotoxic activity of marine algae and a cytotoxic principle of the brown alga Sargassum tortile. Chem. Pharm. Bull.39(8): 2129-2131.

[12] Odaka H, Okimoto Y, Yamada T, Okamoto H, Kawasaki M and Tokura Y (2006) Control of magnetic-field effect on electroluminescence in Alq 3-based organic light emitting diodes. Appl. Phys. Lett.88(12): 123501-123501. 
[13] Popovic, Zoran D and Hany A (2002) Reliability and degradation of small molecule-based organic light-emitting devices (OLEDs). IEEE J. Sel. Top. Quantum Electron. 8(2): 362-371.

[14] Seo YS and Moon DG (2014) Effects of BCP electron transport layer thickness on the efficiency and emission characteristics of white organic light-emitting diodes. J. KIEEME. 27: 45-49.

[15] Shoustikov, Andrei A, Yujian Y and Mark E T (1998) Electroluminescence color tuning by dye doping in organic light-emitting diodes. IEEE J. Sel. Top. Quantum Electron. 4(1): 3-13.

[16] Shimatani K, Tajima H, Komino T, Ikeda S, Matsuda M, Ando Y and Akiyama H (2005) The electroluminescence spectrum of chlorophyll a. Chemi. Let.34(7): 948-949.

[17] Wu CC, Chun JKM, Burrows PE, Sturm JC, Thompson ME, Forrest SR and Register RA (1995) Poly (p-phenylenevinylene)/tris (8-hydroxy) quinoline aluminum heterostructure light emitting diode. Appl. Phys. Lett.66(6): 653-655.
[18] Winter S, Reineke S, Walzer K and Leo K (2008) Photoluminescence degradation of blue OLED emitters. In Photonics Europe.International Society for Optics and Photonics.69992N-69992N.

[19] Wong WY and Ho CL (2009) Functional metallophosphors for effective charge carrier injection/transport: new robust OLED materials with emerging applications. J. Mater. Chem.19(26): 4457-4482.

[20] Wi SG, Kim HJ, Mahadevan SA, Yang DJ and Bae HJ (2009) The potential value of the seaweed Ceylon moss (Gelidium amansii) as an alternative bioenergy resource. Bioresour. Technol.100(24): 6658-6660.

[21] Yersin H (2004) Triplet Emitters for OLED Applications. Mechanisms of Exciton Trapping and Control of Emission Properties. Top. Curr. Chem.242: 1-26.

[22] Zhao YS, Fu H, Peng A, Ma Y, Liao Q and Yao J. (2009). Construction and optoelectronic properties of organic one-dimensional nanostructures. Acc. Chem. Res.43(3): 409-418. 